\title{
Heteroatom Nucleophile Induced C-C Fragmentations to Access Functionalized Allenes
}

\author{
Tanguy Saget ${ }^{\S}$ and Nicolai Cramer ${ }^{\star}$ \\ §SCS-Metrohm Foundation Award for best oral presentation
}

\begin{abstract}
The rich structural and reactivity profile of allenes render them versatile synthetic intermediates. However, application of allenes in organic chemistry is often attenuated by inconvenient methods of preparation. Herein we present an operationally simple route to functionalized allenes from vinyl triflate precursors through a Grob-type fragmentation induced by heteroatom nucleophiles such as water, alcohols, thiols or amines. The fragmentation occurs under mild conditions and is well suited for one-pot or domino processes allowing access to a range of synthetically useful intermediates.
\end{abstract}

Keywords: Allenes · Cyclization · Domino reactions · Fragmentation · Radicals

The allene moiety is a versatile and uniquely reactive functional group which has become popular in modern organic chemistry, especially in transition-metal catalysis. ${ }^{[1]}$ Although several methods for their preparation are established, additional and complementary methods to access them are of synthetic significance. ${ }^{[2]}$ Additionally, the use of mild reaction conditions is highly desirable since the reactive profile of the allene would be well suited for one pot or domino processes. Such an approach would lead to a rapid increase in molecular complexity from rather simple precursors.

The carbonyl-generating Grob fragmentation is a well-established cleaving process which has found widespread application in synthesis. ${ }^{[3]}$ Since the seminal work of Eschenmoser, ${ }^{[4]}$ numerous related reactions have been reported in the literature. Dudley and coworkers studied nucleophile-induced Grob-type fragmentation reactions of vinylogous acyl triflates leading to $\omega$-substituted alkynes. ${ }^{[5]}$ Furthermore, Williams and coworkers recently extended this strategy to access allenes (Scheme

${ }^{\star}$ Correspondence: Prof. Dr. N. Cramer Ecole Polytechnique Fédérale de Lausanne EPFL SB ISIC LCSA

BCH 4305

$\mathrm{CH}-1015$ Lausanne

Tel.: +41216939839

Fax: +41216939700

E-mail: nicolai.cramer@epfl.ch
1). ${ }^{[6]}$ However, their method is limited by the use of hard carbon-nucleophiles such as organolithium or organocerium reagents. Moreover, only products with the carbonyl moiety at the ketone oxidation level or tertiary alcohols resulting from a second addition of the nucleophile are accessible. Reactions induced by heteroatom nucleophiles that would provide versatile carbonyl bearing products at the carboxylic acid oxidation level are not possible with the reported set of reaction conditions.

Our group has a long-standing interest in the development of metal-catalyzed $\mathrm{C}-\mathrm{H}$ bond activations. ${ }^{[7]}$ In this context, we have prepared compounds such as vinyl triflate $\mathbf{1}$ as substrates to develop enantioselective palladium-catalyzed $\mathrm{C}\left(\mathrm{sp}^{3}\right)-\mathrm{H}$ functionalizations. Unexpectedly, 1a was converted to product $\mathbf{6}$ under several reaction conditions we tested. To our surprise, we discovered that a palladium catalyst

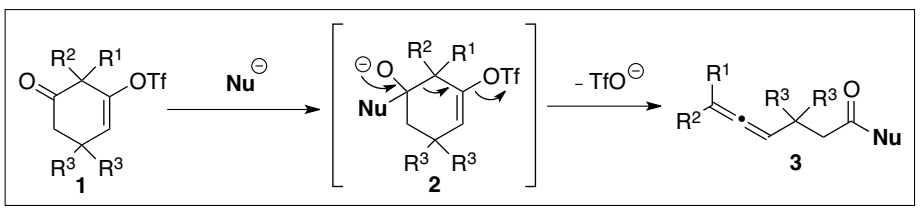

Scheme 1. Nucleophile induced Grobtype fragmentations to access allenes.

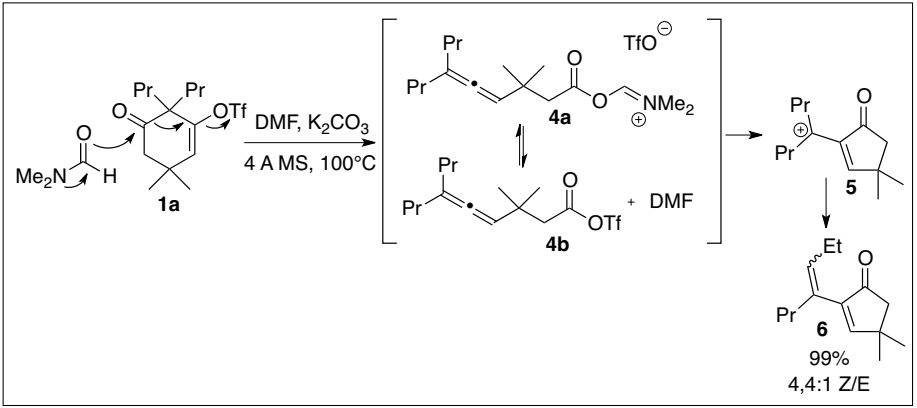

was not required for this transformation. After careful examination of the reaction parameters, we found that $\mathbf{1 a}$ is quantitatively transformed into enone $\mathbf{6}$ when heated in DMF at $100{ }^{\circ} \mathrm{C}$ in the presence of potassium carbonate. ${ }^{[8]}$ One can rationalize the formation of $\mathbf{6}$ by a mechanism which starts by a nucleophile-induced Grob-fragmentation in which DMF acts as the attacking nucleophile. The resulting intermediate $\mathbf{4 a}$ or its acyl triflate equivalent $\mathbf{4 b}$ can subsequently undergo an intramolecular Friedel-Crafts acylation leading to allylic carbenium 5 which gives $\mathbf{6}$ after proton elimination (Scheme 2). After this initial discovery, we decided to follow up this reactivity and to explore the potential of this transformation. First we reasoned that if DMF can promote the fragmentation of $\mathbf{1}$, then other nucleophiles such as alcohols and amines might be competent as well.
Scheme 2. Lead discovery of a domino reaction consisting of a fragmentation and a Friedel-Crafts reaction. 
In this respect, the fragmentation was initially examined with substrate $\mathbf{1 a}$ and benzyl alcohol as the promoter in the presence of a base. We found that the reaction proceeds equally well in polar aprotic solvents such as DMF, DMA or DMSO while nonpolar solvents like toluene or DCE are not competent. The influence of the base was also investigated. Cesium carbonate gave the best results, allowing fast conversions at room temperature. Potassium carbonate displayed somewhat lower reactivity whereas poor conversions were observed with sodium bicarbonate, triethylamine or in the absence of any base. Under the optimized reaction conditions, the desired allene $\mathbf{7 a}$ was obtained in $70 \%$ yield (Scheme 3). Different allenes such as 7b could be synthesized with similar efficiency. The more acidic phenol works even better as shown for 7c,d. Remarkably, the ambident nucleophile 4-aminophenol reacts selectively with the hydroxyl group to give $\mathbf{7 g}$ in good yield. Moreover, water is a powerful promoter and by simply running the reaction in wet solvent, the correspond- ing carboxylic acids $\mathbf{7 e , f}$ were obtained in high yields. Finally, thiophenol is also competent to promote the reaction giving access to valuable thioester $\mathbf{7 h}$, available for further derivatization reactions, e.g. Liebeskind-couplings.

To showcase the utility of this reaction, we studied the lactonization of the carboxylic acid products as the resulting $\gamma$-butyrolactones represent an abundant motif in natural products (Scheme 4). ${ }^{[9]}$ For instance, cyclization to the desired five-membered allylic lactones $8 \mathbf{a}, \mathbf{b}$ occurs in good yields in the presence of trifluoroacetic acid. The reaction proceeds presumably via protonation of the allene. The resulting allylic carbocation is in turn intramolecularly trapped by the carboxylic acid moiety to give $\mathbf{8 a}, \mathbf{b}$. We found that acids 7 also undergo iodolactonizations in a one-pot process from 1 by simply adding iodine to the reaction mixture after completion of the fragmentation step. ${ }^{[10]}$ The resulting vinyl iodides $\mathbf{8 c , d}$ are valuable intermediates which can be further transformed into tetrasubstituted olefins
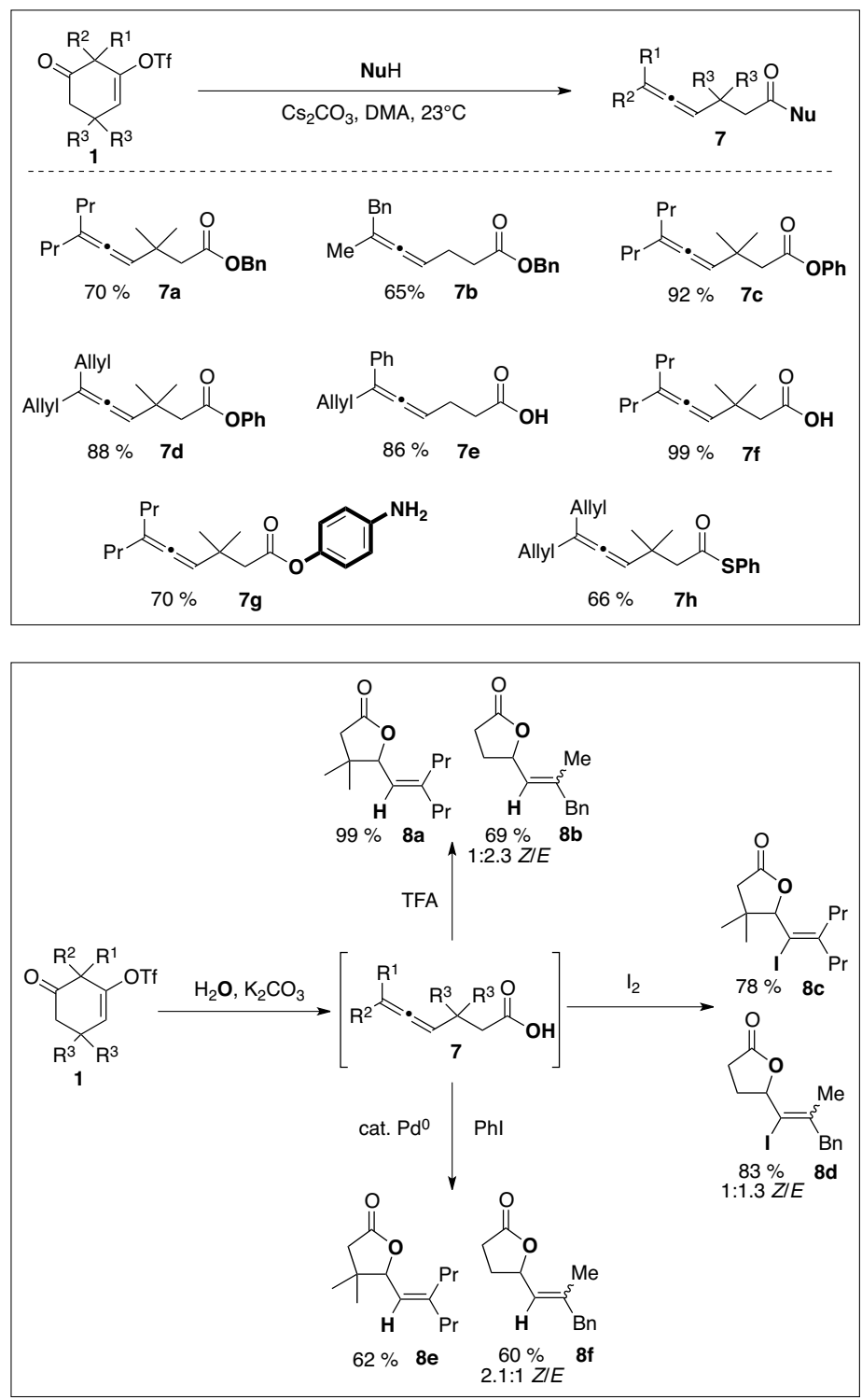

by standard cross-coupling reactions. Alternatively, such tetrasubstituted olefins are directly obtained from allenes 7 through a one-pot fragmentation-arylative cyclization sequence. ${ }^{[11]}$ The addition of a catalytic amount of $\left[\mathrm{Pd}(\mathrm{dba})_{2}\right]$ and tricyclohexyl phosphine with phenyl iodide to allenes 7 induces lactonization to products 8e,f. From a mechanistic point of view, oxidative addition of $\mathrm{Pd}^{0}$ to the aryl iodide generates an $\left[\mathrm{Ar}-\mathrm{Pd}{ }^{\mathrm{II}}-\mathrm{X}\right]$ species which can act as a $\pi$-Lewis acid to activate the allene moiety of $\mathbf{5}$, thus promoting a Wacker-type cyclization followed by a reductive elimination. A second possible mechanism is a carbo-palladation of the allene to form a $\pi$-allyl palladium intermediate which is trapped by an intramolecular nucleophilic attack of the carboxylic acid. ${ }^{[11]}$

We then evaluated nitrogen-containing nucleophiles as a second major class of heteroatom nucleophiles for the fragmentation. We were pleased that they efficiently promote the reaction with only minor changes to the prior established reaction conditions. Both primary and secondary amines lead to allenes $\mathbf{9 a}, \mathbf{b}$ in excellent yields (Scheme 5). Aniline and even free ammonia are also competent nucleophiles (9c,d). Moreover, significantly less nucleophilic sulfonamides can promote the fragmentation provided that cesium carbonate is used as base instead of potassium carbonate. Interestingly, when the fragmentation was conducted with the ambident $N$-phenyl hydroxylamine, the nitrogen atom selectively reacts to afford hydroxamic acid derivative $\mathbf{9 f}$.

Inspired by a recent report on oxidative cyclization of unsaturated hydroxamic acids, ${ }^{[12]}$ 9f seemed to be an ideal precursor for a further cyclization. Heating $9 f$ in acetic acid in the presence of oxygen and lauroyl peroxide at $60{ }^{\circ} \mathrm{C}$ resulted in the formation of 1,2-oxazinone $\mathbf{1 3}$ in fair yield. From a mechanistic point of view, we presume that the initially formed oxygen centered radical $\mathbf{1 0}$ cyclizes to generate a highly reactive vinyl radical species 11, which in turn is trapped by molecular oxygen. The resulting hydroperoxide $\mathbf{1 2}$ rearranges into hydroxyketone product $\mathbf{1 3}$ (Scheme 6). This radical cyclization represents a convenient approach to densely functionalized 1,2-oxazinones which are an important class of heterocycles encountered in several biologically active compounds.

Finally, we turned our attention to sodium azide as another nitrogen-containing promoter for the fragmentation of $\mathbf{1}$ (Scheme 7). With the azide anion as nucleophile, this would lead to the formation of an acyl azide intermediates 14. These derivatives are known to undergo Curtiusrearrangements generating isocyanates $\mathbf{1 5}$. Indeed, heating $1 \mathrm{a}$ in the presence of $\mathrm{NaN}_{3}$ 

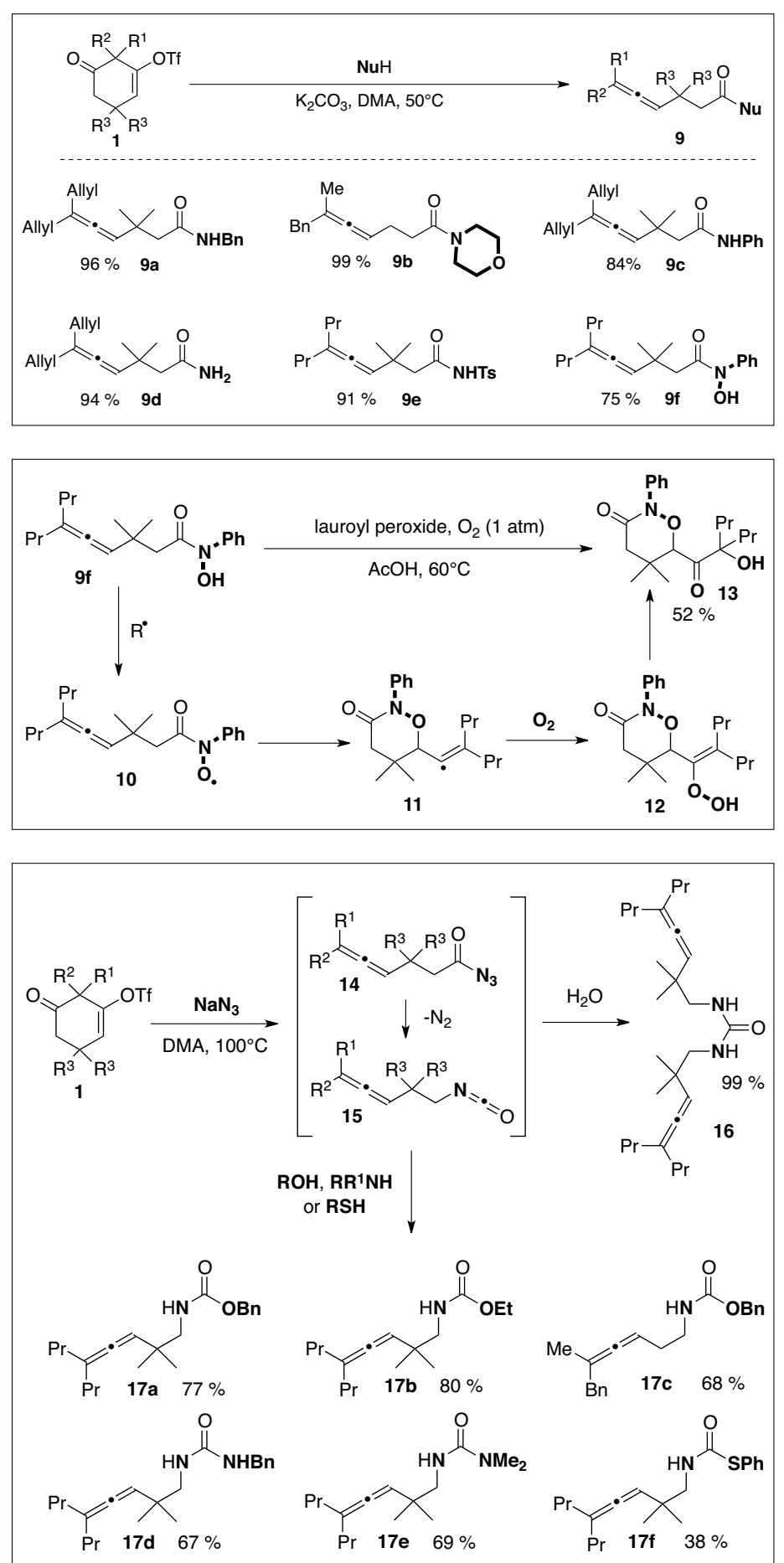

Scheme 7. Domino reaction consisting of the fragmentation and a Curtius rearrange-

ment.

Scheme 6. Oxidative radical cyclization of allenic hydroxamic acid $9 f$.

Scheme 5. Fragmentation induced by nucleophiles.

at $100{ }^{\circ} \mathrm{C}$ in DMF resulted in full conversion to isocyanate 15a which was isolated as the symmetrical urea $\mathbf{1 6}$ upon aqueous work-up. When alcohols are added to the reaction mixture from the beginning, isocyanates $\mathbf{1 5}$ are directly trapped to the corresponding carbamates $\mathbf{1 7 a}, \mathbf{c}$. A similar strategy can be used with amines or thiols leading to ureas $\mathbf{1 7 d}$,e and thiocarbamate 17f. In these cases, the nucleophile has to be added after completion of the fragmentation step.

In conclusion, we have reported a practical and operationally simple synthesis of functionalized allenes from readily available vinyl triflate precursors. The employed reaction conditions are mild and well suited to be coupled to one-pot or domino processes. In this case, one can benefit from the inherent reactivity of allenes which allow transformations of these intermediates into a wide range of synthetically useful compounds.

\section{Acknowledgments}

T. S. would like to cordially thank Metrohm and the Swiss Chemical Society for the SCSMetrohm award. We thank the ETH Zurich (ETH-16 09-3) and EPF Lausanne for funding.

Received: January 13, 2012

[1] a) 'The Chemisty of Allenes', Ed. S. R. Landor, Academic Press, London, 1982; b) 'Modern
Allene Chemistry', Eds. N. Krause, A. S. K. Hashmi, Wiley-VCH, Weinheim, 2004.

[2] a) K. M. Brummond, J. E. Deforrest, Synthesis 2007, 795; b) S. Yu, S. Ma Chem. Commun. 2011, 47, 5384 .

[3] K. Pranz, J. Mulzer, Chem. Rev. 2010, 110 , 3741.

[4] A. Eschenmoser, A. Frey, Helv. Chim. Acta 1952, 35, 1660.

[5] a) S. Kamijo, G. B. Dudley, J. Am. Chem. Soc. 2005, 127, 5028; b) S. Kamijo, G. B. Dudley, Org. Lett. 2006, 8, 175; c) D. M. Jones, S, Kamijo, G. B. Dudley, Synlett 2006, 936; d) S. Kamijo, G. B. Dudley, J. Am. Chem Soc. 2006, 128, 6499; e) S. Kamijo, G. B. Dudley, Tetrahedron Lett. 2006, 47, 5629; f) J. Tummatorn, G. B. Dudley, J. Am. Chem. Soc. 2008, 130, 5050; g) D. M. Jones, M. P. Lisboa, S. Kamijo, G. B. Dudley, J. Org. Chem. 2010, 75,3260 .

[6] R. V. Kolakowski, M. Manpadi, Y. Zhang, T. J. Emge, L. J. Williams, J. Am. Chem. Soc. 2009 , $131,12910$.

[7] a) T. Seiser, O. A. Roth, N. Cramer, Angew. Chem. Int. Ed. 2009, 48, 6320; M. Albicker, N. Cramer, Angew. Chem. Int. Ed. 2009, 48, 9139; D. N. Tran, N. Cramer, Angew. Chem. Int. Ed. 2010, 49, 8181; D. N. Tran, N. Cramer, Angew. Chem. Int. Ed. 2011, 50, 11098; T. Saget, S. J. Lemouzy, N. Cramer, Angew. Chem. Int. Ed. 2012, 51, 2238.

[8] T. Saget, N. Cramer, Angew. Chem. Int. Ed. 2010, $49,8962$.

[9] a) M. S. Maier, D. I. G. Marimon, C. A. Stortz M. T. Adler, J. Nat. Prod. 1999, 62, 1565; b) S.-C. Lee, G. D. Brown, J. Nat. Prod. 1998, 61, 29; c) R. R. A. Kitson, A. Millemaggi, R. J. K. Taylor, Angew. Chem. Int. Ed. 2009, 48, 9426.

[10] X. Jiang, C. Fu, S. Ma, Chem. Eur. J. 2008, 14 , 9656.

[11] R. D. Walkup, L. Guan, M. D. Mosher, S. W. Kim, Y. S. Kim, Synlett 1993, 88.

[12] V. A. Schmidt, E. J. Alexanian, Angew. Chem. Int. Ed. 2010, 49, 4491. 